\title{
Diagnosis and treatment recommendations for pediatric respiratory infection caused by the 2019 novel coronavirus
}

\author{
Zhi-Min Chen ${ }^{1}$. Jun-Fen Fu ${ }^{1} \cdot$ Qiang Shu ${ }^{1} \cdot$ Ying-Hu Chen ${ }^{1}$. Chun-Zhen Hua ${ }^{1} \cdot$ Fu-Bang $\mathrm{Li}^{1} \cdot \mathrm{Ru} \mathrm{Lin}^{1}$ Lan-Fang Tang ${ }^{1}$. \\ Tian-Lin Wang ${ }^{1} \cdot$ Wei Wang $^{1} \cdot$ Ying-Shuo Wang ${ }^{1} \cdot$ Wei-Ze Xu ${ }^{1} \cdot$ Zi-Hao Yang $^{1} \cdot$ Sheng Ye $^{1} \cdot$ Tian-Ming Yuan $^{1}$. \\ Chen-Mei Zhang ${ }^{1} \cdot$ Yuan-Yuan Zhang ${ }^{1}$
}

Received: 2 February 2020 / Accepted: 2 February 2020 / Published online: 5 February 2020

(c) Children's Hospital, Zhejiang University School of Medicine 2020

\begin{abstract}
Since December 2019, an epidemic caused by novel coronavirus (2019-nCoV) infection has occurred unexpectedly in China. As of 8 pm, 31 January 2020, more than 20 pediatric cases have been reported in China. Of these cases, ten patients were identified in Zhejiang Province, with an age of onset ranging from 112 days to 17 years. Following the latest National recommendations for diagnosis and treatment of pneumonia caused by 2019-nCoV (the 4th edition) and current status of clinical practice in Zhejiang Province, recommendations for the diagnosis and treatment of respiratory infection caused by 2019-nCoV for children were drafted by the National Clinical Research Center for Child Health, the National Children's Regional Medical Center, Children's Hospital, Zhejiang University School of Medicine to further standardize the protocol for diagnosis and treatment of respiratory infection in children caused by $2019-n \mathrm{CoV}$.
\end{abstract}

Keywords New coronavirus $\cdot$ Respiratory infection $\cdot$ Child $\cdot$ Diagnosis $\cdot$ Treatment $\cdot$ Recommendation

\section{Background}

Since December 2019, an epidemic caused by novel coronavirus (2019-nCoV) infection has occurred unexpectedly in China. At present, 2019-nCoV infection has been a legal class B infectious disease of the Law of the People's Republic of China on the Prevention and Treatment of Infectious Diseases, and managed as a class A infectious disease for infection prevention and control practices.

As of $8 \mathrm{pm}, 31$ January, more than 20 pediatric cases have been reported in China. Of these cases, ten were identified in Zhejiang Province, with an age of onset ranging from 112 days to 17 years. Following latest National recommendations for diagnosis and treatment of respiratory infections

Jun-Fen Fu

fjf68@zju.edu.cn

Qiang Shu

shuqiang@zju.edu.cn

1 Children's Hospital, Zhejiang University School of Medicine, National Clinical Research Center for Child Health, National Children's Regional Medical Center, Hangzhou 310052, China caused by 2019-nCoV (the 4th edition) and current status of clinical practice in Zhejiang Province, recommendations for the diagnosis and treatment of respiratory infection caused by $2019-\mathrm{nCoV}$ for children were drafted out by the National Clinical Research Center for Child Health, National Children's Regional Medical Center, Children's Hospital, Zhejiang University School of Medicine to further standardize the protocol of respiratory infection in children caused by 2019-nCoV.

\section{Etiology}

2019-nCoV is a novel human coronavirus in addition to coronavirus 229E, NL63, OC43, HKU1, Middle East respiratory syndrome-related coronavirus (MERSr-CoV) and severe acute respiratory syndrome-related coronavirus (SARS-CoV). 2019-nCoV is enveloped single-stranded plus stranded RNA virus with a diameter of $60-140 \mathrm{~nm}$, spherical or elliptical in shape and pleomorphic [1]. It has been reported that the consistency of whole genome-wide nucleotide sequences of 2019-nCoV with SARS-like coronavirus in bats (bat-SL-CoVZC45) ranges from 86.9 [1] to $89 \%$ [2]. The nucleotide sequence of spines protein on the 
envelope of the virus is also highly consistent with that of bat-SL-CoVZC45 (84\%) and SARS-CoV (78\%).

The physicochemical property of 2019-nCoV has not been clarified clearly yet. It is thought that $2019-\mathrm{nCoV}$ is sensitive to ultraviolet radiation and heating. For example, according to researches on SARS-CoV and MERS-COV, the virus can be inactivated by heating at $56^{\circ} \mathrm{C}$ for 30 minutes and by using lipid solvents such as $75 \%$ ethanol, chlorinecontaining disinfectant, peroxyacetic acid and chloroform, but not by chlorhexidine, according to the researches on SARS-CoV and MERS-CoV.

\section{Epidemiology}

\section{Infection sources}

The main sources of the infection are patients infected by 2019-nCoV with or without clinical symptoms [2]. In addition, patients in the incubation period may also have potency to transmit the virus based on the case evidence.

\section{Transmission route}

The novel virus is spread through respiratory droplets when patients cough, talk loudly or sneeze. Close contact is also a source of transmission (e.g., contact with the mouth, nose or eye conjunctiva through contaminated hand). Whether transmission can occur through mother-infant vertically or breast milk has not been established yet.

\section{Susceptible population}

There are general susceptibilities in all groups, with the elderly and people with basic diseases more likely to become severe cases. Children may have mild clinical symptoms after infection [3].

\section{Clinical characteristics}

\section{Clinical presentations}

The incubation period of 2019-nCoV infections ranges from 2 to 14 days, though most often rangs from 3 to 7 days [3].

At the onset of the disease, infected children mainly present with fever, fatigue and cough, which may be accompanied by nasal congestion, runny nose, expectoration, diarrhea, headache, etc. Most of the children had low to moderate fever, even no fever. Dyspnea, cyanosis and other symptoms can occur as the condition progresses usually after 1 week of the disease, accompanied by systemic toxic symptoms, such as malaise or restlessness, poor feeding, bad appetite and less activity. The condition of some children may progress rapidly and may develop into respiratory failure that cannot be corrected by conventional oxygen (nasal catheter, mask) within 1-3 days. In these severe cases, even septic shock, metabolic acidosis and irreversible bleeding and coagulation dysfunction may occur.

Rapid respiration rate and moist rales on auscultation usually indicate pneumonia. The criteria for rapid respiratory rate are as follows: $\geq 60$ times/min for less than 2 months old; $\geq 50$ times/min for $2-12$ months old, $\geq 40$ times/min for $1-5$ years old, $\geq 30$ times/min for $>5$ years old (after ruling out the effects of fever and crying). With the aggravation of the disease, respiratory distress, nasal flaring, suprasternal, intercostal and subcostal retractions, grunting and cyanosis may occur.

Maternal hypoxemia caused by severe infection can lead to intrauterine asphyxia, premature delivery and other risks. Neonates, especially preterm infants, who are more likely to present with insidious and non-specific symptoms need more close observation.

According to the current conditions of the reported cases, the children mainly belong to family cluster cases. Most of them have good prognosis, and in mild cases recover 1-2 weeks after disease onset. No deaths in children have been reported up to now.

\section{Laboratory examinations [3]}

Routine blood test White blood cell count is usually normal or reduced, with decreased lymphocyte count; progressive lymphocytopenia in severe cases.

$C$-reactive protein $(C R P)$ normal or increased.

Procalcitonin (PCT) Normal in most cases. The level of PCT $>0.5 \mathrm{ng} / \mathrm{mL}$ indicates the co-infection with bacteria.

Others Elevation of liver enzymes, muscle enzymes and myoglobin, and increased level of D-dimer might be seen in severe cases.

\section{Etiologic detection}

Nucleic acid testing is the main method of laboratory diagnosis. 2019-nCoV nucleic acid can be detected by RT-PCR or by viral gene sequencing of throat swabs, sputum, stool or blood samples.

Other methods 2019-nCoV particles can be isolated from human respiratory epithelial cells through virus culture [4, 5], but this experiment cannot be carried out in general laboratories. Virus antigen or serological antibody testing kits are not available at present.

\section{Imaging features [3]}

Chest X-ray examination In the early stage of pneumonia cases, chest images show multiple small patchy shadows 
and interstitial changes [4], remarkable in the lung periphery [2]. Severe cases can further develop to bilateral multiple ground-glass opacity, infiltrating shadows, and pulmonary consolidation, with infrequent pleural effusion.

Chest CT scan Pulmonary lesions are shown more clearly by $\mathrm{CT}$, including ground-glass opacity and segmental consolidation in bilateral lungs, especially in the lung periphery. In children with severe infection, multiple lobar lesions may be present in both lungs.

\section{Diagnostic criteria}

\section{Suspected cases}

Patients should be suspected of 2019-nCoV infection who if they meet any one of the criteria in the epidemiological history and any two of the criteria in clinical manifestations.

\section{Epidemiological history}

1. Children with a travel or residence history in Wuhan City and neighboring areas, or other areas with persistent local transmission within 14 days prior to disease onset.

2. Children with a history of contacting patients with fever or respiratory symptoms who have a travel or residence history in Wuhan City and neighboring areas, or in other areas with persistent local transmission within 14 days prior to disease onset.

3. Children with a history of contacting confirmed or suspected cases infected with 2019-nCoV within 14 days prior to disease onset.

4. Children who are related with a cluster outbreak: in addition to this patient, there are other patients with fever or respiratory symptoms, including suspected or confirmed cases infected with 2019-nCoV.

5. Newborns delivered by suspected or confirmed 2019-nCoV-infected mothers.

\section{Clinical presentations}

1. Fever, fatigue, dry cough; some pediatric patients may have no fever.

2. Patients with the above-mentioned chest imaging findings (refer to the section of imaging features);

3. In the early phase of the disease, white blood cell counts are normal or decreased, or with decreased lymphocyte count.

\section{Confirmed cases}

Suspected cases who meet any one of the following criteria:
1. Throat swab, sputum, stool, or blood samples tested positive for 2019-nCoV nucleic acid using RT-PCR;

2. Genetic sequencing of throat swab, sputum, stool, or blood samples being highly homologous with the known 2019-nCoV;

3. 2019-nCoV granules being isolated by culture from throat swab, sputum, stool, or blood samples.

It is necessary to strengthen the awareness of the early identification of the disease. In clinic, screening was conducted mainly according to the epidemiological history and fever or respiratory symptoms, and pathogen examination should be carried out on time. Furthermore, effective isolation measures and appropriate treatment need to be provided promptly. Even if the common respiratory pathogen tests are positive, children who have a history of close contact with 2019-nCoV-infected cases also are recommended for timely 2019-nCoV pathogen testing.

\section{Clinical classifications}

\section{Mild type}

This type of patient includes those with asymptomatic infection, upper respiratory infection (URI) and mild pneumonia. Symptoms include fever, cough, sore throat, fatigue, headache or myalgia. Some patients show pneumonia signs on chest imaging. These patients do not have any of the severe or critical symptoms and complications described below.

\section{Severe pneumonia}

Disease progresses to meet any of the following conditions [6]:

1. Significantly increased respiration rate: $R R \geq 70 / \mathrm{min}$ ( $\leq 1$ year), $\mathrm{RR} \geq 50 / \mathrm{min}$ ( $>1$ year).

2. Hypoxia: $\mathrm{SpO}_{2} \leq 93 \%$ ( $<90 \%$ in premature infants) or nasal flaring, suprasternal, intercostal and subcostal retractions, grunting and cyanosis, apnea, etc.

3. Blood gas analysis: $\mathrm{PaO}_{2}<60 \mathrm{mmHg}$, $\mathrm{PaCO}_{2}>50 \mathrm{mmHg}$.

4. Consciousness disorders: restlessness, lethargy, coma, convulsion, etc.

5. Poor feeding, bad appetite, and even dehydration.

6. Other manifestations: coagulation disorders (prolonged prothrombin time and elevated level of D-dimer), myocardial damage (increased level of myocardial enzyme, electrocardiogram ST-T changes, cardiomegaly and cardiac insufficiency in severe cases), gastrointestinal dysfunction, raised level of liver enzyme and rhabdomyolysis. 


\section{Critical cases}

Disease progresses rapidly along with organ failure with any of the following conditions:

1. Respiratory failure which requires mechanical ventilation Patients present with acute respiratory distress syndrome (ARDS) and are featured by refractory hypoxemia, which cannot be alleviated by conventional oxygen therapy, such as nasal catheter or mask oxygen supplement.

2. Septic shock In addition to severe pulmonary infection, 2019-nCoV can cause damage and dysfunction of other organs. When dysfunction of extrapulmonary system such as circulation, blood and digestive system occurs, the possibility of sepsis and septic shock should be considered and the mortality rate increases significantly.

3. Accompanied by other organ failure that needs ICU monitoring and treatment.

\section{Differential diagnosis}

\section{Other viral respiratory infections}

Viruses such as SARS virus, influenza virus, parainfluenza virus, adenovirus, respiratory syncytial virus and metapneumovirus can cause viral respiratory infections. Patients commonly present with fever, cough and dyspnea, and interstitial pneumonia in some cases. Most of the cases have normal or decreased white blood cell counts, while severely infected children show reduced level of lymphocyte count. Similar to 2019-nCoV, all of these viruses can transmit through respiratory tract or direct contact, which is characterized by clustering onset. Epidemiological exposure history plays an important role in differential diagnosis, which is mainly confirmed by laboratory examinations.

\section{Bacterial pneumonia}

Patients with bacterial pneumonia mostly display high fever and toxic appearance. In the early stage of the disease, cough is not obvious but moist rale can be heard. Chest image may show small patchy shadows, or segmental or even lobar consolidation. Blood routine examination shows increased white blood cell count with neutrophil predominating, as well as elevated CRP. Noninvasive pneumonia is usually accompanied by obvious cough. Antibiotics are effective. Blood or deep sputum culture is helpful for diagnosis of bacterial pneumonia.

\section{Mycoplasmal pneumonia}

Mycoplasmal pneumonia may occur in any season and in schools and child-care institutions with small epidemic. Patients are predominantly school-age children, but the number of younger children is increasing. They usually start with high fever and cough. Chest images may reveal manifestations including reticular shadows and small patchy or large consolidation. Blood routine examination shows that the white blood cell count is normal or increased and CRP is slightly elevated. Nucleic acid detection of airway secretion and serum mycoplasma-specific IgM determination are helpful for differential diagnosis.

It should be noted that patients with 2019-nCoV infection can have co-infection or superimposed infection with other viruses or bacteria simultaneously.

\section{Management principles}

The four principles of "early identification", "early isolation", "early diagnosis", and "early treatment" should be emphasized.

\section{Strict isolation strategies}

Medical isolation is supposed to be carried out once the suspected cases are identified. The confirmed cases should be admitted to the designated hospitals.

\section{Mild cases}

Avoid using broad-spectrum antibiotics and corticosteroids.

\section{Severe and critical case}

Antibiotics, corticosteroids, bronchoalveolar lavage, mechanical ventilation, and other more invasive intervention, such as blood purification and extracorporeal membrane oxygenation (EMCO) should be applied cautiously, based on cost-benefit evaluation.

\section{Multidisciplinary cooperation}

Monitoring patient's conditions closely and adjusting the therapeutic protocols timely through multidisciplinary cooperation are of great significance.

\section{Treatments}

\section{Medical isolation}

Suspected case should be isolated in a single room, while confirmed cases can be arranged in the same room. 


\section{Assessment}

During the course of treatment, pay close attention to the changes in children's conditions, regularly monitor vital signs, $\mathrm{SpO}_{2}$, etc., and identify the severe and critical cases as early as possible.

\section{General treatments}

The general strategies include bed rest and supportive treatments; ensuring sufficient calorie and water intake; maintaining water electrolyte balance and homeostasis, and strengthening psychotherapy for elder children when necessary.

\section{Antiviral therapy}

There are no effective antiviral drugs for children at present. Interferon- $\alpha 2 b$ nebulization can be applied, and the recommended usage is as follows:

1. Interferon- $\alpha 2 b$ nebulization $100,000-200,000 \mathrm{IU} / \mathrm{kg}$ for mild cases, and 200,000-400,000 IU/kg for severe cases, two times/day for 5-7 days.

2. Lopinavir/litonavir (200 $\mathrm{mg} / 50 \mathrm{mg})$ The recommended doses: weight 7-15 kg, $12 \mathrm{mg} / 3 \mathrm{mg} / \mathrm{kg}$; weight $15-40 \mathrm{~kg}, 10 \mathrm{mg} / 2.5 \mathrm{mg} / \mathrm{kg}$; weight $>40 \mathrm{~kg}$, $400 \mathrm{mg} / 100 \mathrm{mg}$ as adult each time, twice a day for 1-2 weeks [3, 7, 8]. However, the efficacy, treatment course and safety of the above drugs remain to be determined.

\section{Antibiotics application}

Irrational use of antibiotics should be avoided. Bacteriological monitoring should be strengthened. If there is evidence of secondary bacterial infection, appropriate antibiotics should be used timely.

\section{Immunomodulating therapy}

Corticosteroids should be avoided in common type of infection. However, it can be considered in the following situations:

1. With rapidly deteriorating chest imaging and occurence of ARDS.

2. With obvious toxic symptoms, encephalitis or encephalopathy, hemophagocytic syndrome and other serious complications.

3. With septic shock.
4. With obvious wheezing symptoms.

Intravenous methylprednisolone ( $1-2 \mathrm{mg} / \mathrm{kg} / \mathrm{day})$ is recommended for 3-5 days, but not for long-term use [3, 5, 9-11].

Intravenous immunoglobulin can be used in severe cases when indicated, but its efficacy needs further evaluation. The recommended dose is $1.0 \mathrm{~g} / \mathrm{kg} /$ day for 2 days, or $400 \mathrm{mg} / \mathrm{kg}$ / day for 5 days [6, 9, 12, 13].

\section{Bronchoalveolar lavage (BAL)}

BAL is not suitable for most patients, and there is an increased risk of cross-infection. The indications should be strictly controlled. BAL can be considered if patients have obvious symptoms of airway obstruction, refractory massive atelectasis indicated by imaging, a significant increase in peak pressure during ventilator therapy, decrease of tidal volume, or poor oxygenation which cannot be reversed by conservative treatments.

\section{Organ function support}

In case of circulation dysfunction, vasoactive drugs should be used to improve microcirculation on the basis of adequate liquid support [3]. Patients with acute renal injury should be given continuous blood purification on time. In the meantime, paying attention to the brain function monitoring if neccessary. If intracranial hypertension and convulsion occurs in children, it is necessary to reduce the intracranial pressure and control convulsion timely $[6,12,13]$.

\section{Respiratory support}

In the case of respiratory distress that occurs despite nasal catheter or mask oxygenation, heated humidified high-flow nasal cannula (HHHFNC), non-invasive ventilation such as continuous positive airway pressure (CPAP), or non-invasive high-frequency ventilation can be used. If improvement is not possible, mechanical ventilation with endotracheal intubation and a protective lung ventilation strategy should be adopted.

\section{Blood purification}

Continuous blood purification should be considered in cases of multiple organ failure (especially acute kidney injury), or capacity overload and life-threatening imbalance of water, electrolyte, and acid-base. The therapeutic model may include continuous veno-venous hemofiltration (CVVH), continuous veno-venous hemodiafiltration (CVVHDF), or heterozygosis. If combined with liver failure, plasma exchange is feasible. 


\section{Extracorporeal membrane oxygenation (ECMO)}

ECMO therapy should be considered when mechanical ventilation, blood purification, and other means are ineffective, and cardiopulmonary failure occurs which is difficult to correct.

1. ECMO indications [14].

2. $\mathrm{PaO}_{2} / \mathrm{FiO}_{2}<50 \mathrm{mmHg}$ or oxygen index $(\mathrm{OI})>40$ for more than $6 \mathrm{~h}$, or severe respiratory acidosis $(\mathrm{pH}<7.15)$.

3. High mean airway pressure (MAP) during mechanical ventilation, or severe air leakage and other severe complications.

4. Circulation function cannot be improved with conventional treatment, or large amounts of vasoactive drugs are required to maintain basal blood pressure, or lactic acid levels continuously rise.

Contraindication [14] ECMO should be contraindicated or used with caution if the duration of mechanical ventilation is more than 2 weeks, or if serious brain failure or bleeding tendency occurs.

\section{Discharge criteria}

Children with body temperature back to normal for at least 3 days, significant improvement in respiratory symptoms, and completion of two consecutive negative tests of respiratory pathogenic nucleic acid (sampling interval of at least 1 day) can be discharged. If necessary, home isolation for 14 days is suggested after discharge.

\section{Principle of transportation}

Special vehicles should be used to transfer infected patients. Strict protection for staff of transportation and disinfection for the vehicle are of vital importance. Enforcing close to the lines of notification of the project for transportation of pneumonia cases infected with novel coronavirus (trial version) published by the National Health Commission of People's Republic of China is required.

\section{Infection control in hospital}

\section{Strict implementation of standard prevention}

Medical personnel are supposed to have good personal protection, hand hygiene, ward management, environmental ventilation, object surface cleaning and disinfection, medical waste management and other hospital infection control work, based on the standard prevention protocol, to minimize nosocomial infection.
Personal protective equipment

1. All medical personnel are required to wear surgical masks during medical activities.

2. Pre-check triage: wearing medical overalls, caps, and surgical masks.

3. Fever clinic, respiratory clinic, emergency department, infectious diseases department, and isolation ward: equipped with medical overalls, caps, disposable isolation clothing, surgical masks, and goggles or face shield for daily medical activities and ward rounds; fit with goggles or face shield is stressed when collecting respiratory samples; using latex gloves in addition when contacting with blood, body fluid, secreta, or excreta; wearing surgical masks, goggles or face shield, latex gloves, medical protective clothing (disposable impermeable isolation clothing can be added), and respiratory hood when necessary, to prevent aerosol or splash during operations of endotracheal intubation, bronchoscopy, airway nursing, and sputum suction.

4. Medical personnel should wear and remove personal protective equipment in strict accordance with the onoff procedure, rather than leaving ward with the contaminated equipment, so as to avoid cross-contamination in different zones.

5. Patients and their accompanying family members are required to wear surgical masks.

\section{Additional notes}

1. Channels for medical personnel and patients in the isolation ward should be separated and equipped with medical personnel channel buffer zone.

2. Wearing gloves cannot replace hand hygiene.

3. Perform strict visiting regulations for pediatric patients admitted in isolation, and ask visitors to have personal protection according to relevant regulations when necessary.

4. Optimize medical procedures to reduce the frequency of medical personnel's contact with patients.

5. Attention should be paid to the strict elimination and disinfection of the patient's secretions and excretions.

Author contributions All authors drafted part of the manuscript and approved the final version.

Funding None.

\section{Compliance with ethical standards}

Ethical approval None. 
Conflict of interest All authors declared no conflict of interest related to this paper.

\section{References}

1. Zhu N, Zhang D, Wang W, Li X, Yang B, Song J, Zhao X, Huang B, Shi W, Lu R, Niu P, Zhan F, Ma X, Wang D, Xu W, Wu G, Gao GF, Tan W, Coronavirus Investigating, and Research Team. A novel coronavirus from patients with pneumonia in China, 2019. N Engl J Med. 2020. https://doi.org/10.1056/NEJMoa2001 017.

2. Chan JF, Yuan S, Kok KH, To KK, Chu H, Yang J, Xing F, Liu J, Yip CC, Poon RW, Tsoi HW, Lo SK, Chan KH, Poon VK, Chan WM, Ip JD, Cai JP, Cheng VC, Chen H, Hui CK, Yuen KY. A familial cluster of pneumonia associated with the 2019 novel coronavirus indicating person-to-person transmission: a study of a family cluster. Lancet. 2020. https://doi.org/10.1016/S0140 -6736(20)30154-9.

3. National Recommendations for Diagnosis and Treatment of pneumonia caused by 2019-nCoV (the 4th edition). National Health Commission and National Administrative Office of Chinese Tradition Medicine. https://www.nhc.gov.cn/xcs/zhengcwj/20200 1/4294563ed35b43209b31739bd0785e67/files/7a9309111267475 a99d4306962c8bf78.pdf. Access 29 Jan 2020.

4. Huang C, Wang Y, Li X, Ren L, Zhao J, Hu Y, et al. Clinical features of patients infected with 2019 novel coronavirus in Wuhan, China. Lancet. 2020. https://doi.org/10.1016/S0140 -6736(20)30183-5

5. Stanley P. Another decade, another coronavirus. N Engl J Med. 2019. https://doi.org/10.1056/NEJMe2001126.

6. Ma XJ, Wang LN, Wang LB, Wang XF, Fang F, Deng L, et al. Diangosis and treatment guideline of community acquired pneumonia in children. Chin Clin Infect Dis J. 2019;12:6-13.

7. Chu CM. Role of Lopinavir/ritonavir in the treatment of SARS: initial virological and clinical findings. Thorax. 2004;59:252-6.
8. Arabi YM, Alothman A, Balkhy HH, Al-Dawood A, AlJohani S, Al Harbi S, et al. Treatment of Middle East respiratory syndrome with a combination of lopinavir-ritonavir and interferon$\beta 1 \mathrm{~b}$ (MIRACLE trial): study protocol for a randomized controlled trial. Trials. 2018;19:81.

9. Lu Q, Wang XF, Qiang Y, Li XM, Zhang HL, Wang LN, et al. Expert consensus on the diagnosis and treatment of viral pneumonia in children (2019). Zhongguo Shi Yong Er Ke Za Zhi. 2019;34:801-7 (in Chinese).

10. Arabi YM, Mandourah Y, Al-Hameed F, Sindi AA, Almekhlafi GA, Hussein MA, et al. Corticosteroid therapy for critically ill patients with Middle East respiratory syndrome. Am J Respir Crit Care Med. 2018;197:757-67.

11. WHO. Clinical management of severe acute respiratory infection when novel coronavirus (nCoV) infection is suspected. Jan 11, 2020. https://www.who.int/docs/default-source/coronaviruse/clini cal-management-of-novel-cov.pdf?sfvrsn=bc7da517_2. Accessed 30 Jan 302020.

12. The Subspecialty Group of Respiratory Diseases, The Editorial Board, Chinese Journal of Pediatrics. Diagnosis and treatment guideline of community acquired pneumonia in children (2013 the First part). Zhonghua Er Ke Za Zhi. 2013;51:745-52.

13. Wang XF, Deng L, Liu JR, Liu CF, Liu EM, Liu HW, et al. Guidelines for the diagnosis and treatment of adenovirus pneumonia in children (2019 edition). Chin J Cli Infect Dis. 2019;12:161-6 (in Chinese).

14. Pediatric Group, Extracorporeal Life Support Committee, Pediatric Critical Physicians Branch, Chinese Medical Association Emergency Group, Pediatric Branch, Chinese Medical Association Thoracic Vascular Anesthesiology Society, Extracorporeal Life Support Committee, Pediatric Surgery Branch, Chinese Medical Association. Consensus of extracorporeal membrane oxygenation support for acute fulminant myocarditis in children. Chin J Emerg Med. 2020;29:5-10 (in Chinese).

Publisher's Note Springer Nature remains neutral with regard to jurisdictional claims in published maps and institutional affiliations. 